\title{
Safety of users in road evacuation: pedestrian outflow models in a building
}

\author{
M. Di Gangi ${ }^{1} \&$ P. Velonà ${ }^{2}$ \\ ${ }^{1}$ Department of Architecture, Planning and Transport Infrastructure, \\ University of Basilicata, Italy \\ ${ }^{2}$ Department of Computer Science, Mathematics, Electronics and \\ Transportation, Mediterranea University of Reggio Calabria, Italy
}

\begin{abstract}
In this paper we present some results obtained in the research project entitled SURE, carried out by the Laboratory for Transport Systems Analysis (LAST) of the Mediterranea University of Reggio Calabria (Italy).

The general objective of the project is risk reduction in urban areas in terms of exposure through the definition and implementation of evacuation procedures. One of the activities concerns the specification and calibration of a system of models able to simulate the transportation system when a population has to evacuate due to a forthcoming disaster.

This paper concerns the simulation of pedestrian outflow related to the evacuation of a building, using flow models calibrated on the basis of data collected during experimentation on a test site. This experimentation was conducted in a school in an Italian town but the methodology used can be applied to any building with homogeneous characteristics in terms of activities (i.e. offices, banks, commercials, schools).
\end{abstract}

\section{Introduction}

In ordinary conditions for simulating pedestrian flow two approaches have been proposed: the first approach [1] considers the speed-density function; the second approach considers the level of services [2]. In these studies models are calibrated referring to user categories. In [1] both ordinary and emergency conditions are taken into consideration and a similar approach is also used in [3]. 
In [2], [4] and [5], the level of service approach is considered. Recently, due to natural disasters and terrorist attacks, attention to problems connected to both pedestrian and motorized evacuation has broadened and many international conferences have devoted specific sessions to topics concerning problems arising during evacuation. As an example, pedestrian evacuation, for different sites of application, is the subject of many contributions contained in [6].

In this paper evacuation from a school is simulated using a dynamic model of network loading. The outflow characteristics depend on the conditions taking place on the network links, through which the transport supply is represented, and vary temporally in a discrete way; that allows us both to estimate the evacuation time and to analyse the temporal course of the outflow conditions throughout the event. Thus, in order to reproduce these phenomena, it is necessary either to use static models in pseudo-dynamic assignment procedures [7-9], or to remove the intra-period stationarity hypothesis and choose withinday dynamic assignment models (Dynamic Traffic Assignment models - DTA).

In general, DTA models can be classified according to the representation of traffic variables (continuous or discrete) or the nature of the variables representing network performances (aggregate or disaggregate). So-called macroscopic models (or Flow-based Analytical Models: [10-16]), simulate network performances by means of aggregate variables (speed, density, flow) with explicit capacity, as in static models, and use a continuous representation of traffic; generally, in the formulation of macroscopic models, fluid-dynamic analogies of traffic are adopted.

A second type of model, named mesoscopic (or Packet-based models: [1722]), is similar to the previous one in the way it simulates network performances (aggregate variables with explicit capacity are used), but it differs in terms of traffic representation; the peculiarity of mesoscopic models consists of a discrete flow representation for groups of vehicles (users).

A third type is made up by microscopic models, where individual trajectories of all the vehicles or users are simulated by using disaggregate variables with implicit capacity, and a discrete traffic representation.

Within the dynamic loading model, the need to take account of the very particular conditions occurring during evacuation yielded to the specification and calibration of specific outflow functions.

For the reasons stated above, the analysis was developed in the following phases:

- model descriptions, where the structure of models adopted in the following application phase is proposed;

- real experimentation, divided into:

1 on site experimentation; concerning a simulation carried out in the town of Melito Porto Salvo (Reggio Calabria, Italy) consisting in the evacuation of a primary school;

1 model calibration, during which outflow functions, related to particular structural elements of the building (stairs, corridors, doors), were specified and calibrated; 
- $\quad$ application, during which analysis of the evacuation from the building was carried out by performing a computer simulation using the outflow functions previously specified and calibrated within the adopted dynamic model of network loading.

The results obtained during the computer simulation performed in the application phase were compared with those obtained during the experimentation phase.

This paper is structured as follows: in chapter 2 the system of models used in the application phase is described, chapter 3 reports operations carried out during on site experimentation and the calibration of models, and chapter 4 presents an analysis of results obtained within the application and a comparison between these results and on site experimentation data.

\section{Models}

\subsection{Supply model}

Two elements are considered within the supply model:

- the topological representation of the pedestrian network;

- the representation of outflow conditions by means of specific relationships. The pedestrian network is represented using fundamentals of graph theory. The classes of components making up the graphs are:

- $\quad$ area centroids: these nodes represent the barycentre of each area (rooms, offices, etc.) comprising the building. They sum up the origins of the trips of all the people who, at emergency time, are within the area considered. There is a centroid for each area in which the building is divided;

- destination nodes (centroids): these represent the safe areas towards which people converge in an emergency. Thus they correspond to the destination of each path;

- $\quad$ network nodes: these are located at each potential change of direction along a generic footpath or at significant variations in geometric and/or functional characteristics of a trunk (i.e. width variations);

- real arcs: these represent the connection between two network nodes or a network and a destination node; they coincide with trunks of the pedestrian network and are classified into flat ramps (corridors) and descending ramps (stairs);

- connector arcs: these represent the connection between a centroid and a network node.

The representation of outflow conditions requires that specific relationships among variables be defined. In this particular case functional dependence of speed with density was investigated.

A model able to reproduce outflow speed in descending flights of a stair depending on density and of geometrical characteristics was formulated as:

$$
v=a \cdot A / P+b \cdot(\delta)^{c}+d \cdot L+e \cdot I / E+f \cdot N G+g[\mathrm{~m} / \mathrm{s}]
$$


where:

$v$ outflow speed $(\mathrm{m} / \mathrm{s})$;

$A$ raised of the step;

$P$ depth of the step;

$\delta$ density (users $/ \mathrm{m}^{2}$ );

$L$ width (m);

$I / E$ dummy variable $=1$ if inner stairs; $=0$ otherwise;

$N G$ number of steps;

$a, b, c, d, e, f, g \quad$ parameters to be calibrated.

Boarding on bus was also considered, taking account of the peculiarities of most users considered (primary school pupils). Several specifications were first considered:

where:

$$
\begin{aligned}
& T b=r+s \cdot N / n_{d} \\
& T b=r+\left(N / n_{d}\right)^{s} \\
& T b=r+\exp \left\{s \cdot N / n_{d}\right\}
\end{aligned}
$$

$T b$ boarding time (sec);

$N$ number of boarding people;

$n_{d}$ number of doors;

$r, s$ parameters to be calibrated.

\subsection{Demand model}

On the basis of the location of the safe areas defined by the existing evacuation plan, the building in question is included in an influence zone corresponding to a safe area of destination. The level of demand can be estimated separately for different categories (i.e. residents within the area; non-residents who systematically reach the area for work; non-residents who occasionally reach the area for shopping or other activities). In the particular case considered here both pupils attending lessons and staff working in the school were considered.

In order to allow for the different distribution of population within the area, different hypotheses should be considered depending on the time of the evacuation defined for the reference scenario (i.e. day of the week, hour of the day).

\subsection{Demand - supply interaction model}

Static models do not allow analysis of phenomena connected to temporal variations in terms of both demand and supply, such as rising and scattering of queues due to temporary peaks of demand and/or capacity reductions of infrastructures.

The Dynamic Traffic Assignment (DTA) model considered here to simulate evacuation is mesoscopic and is based on that proposed by Cascetta and Cantarella [21] and subsequently developed [22-24], where users are assembled 
in packets that move on the network discretising demand for each origindestination pair. The model described here consists of an evolution of the dynamic approach developed for evacuation purposes in [25], and applied in [26] for ship evacuation and in [27] as a support for the design of evacuation plans.

\section{Experimentation}

\subsection{The test site}

A description of the whole test site of the town of Melito Porto Salvo (Reggio Calabria, Italy) can be found in another paper [28] published in this volume. The building in question is a primary school located within the CBD area selected for the drill. The school has its own evacuation plan which, for the sake of this drill, was correlated to the evacuation plan of the whole town. The school evacuation plan stipulates that everybody must gather at a site in front of the building (called first assembly point below); according to the town evacuation plan, the school's staff and pupils will be led to the safe place on a bus service starting from another gathering place (second assembly point below) that must be reached in order to evacuate the area completely (see Fig. 1). Hence evacuation of the school was schematized in following four main phases:

1. evacuation of the building reaching first assembly point;

2. roll-call of pupils at first assembly point;

3. transfer to second assembly point;

4. boarding on bus.

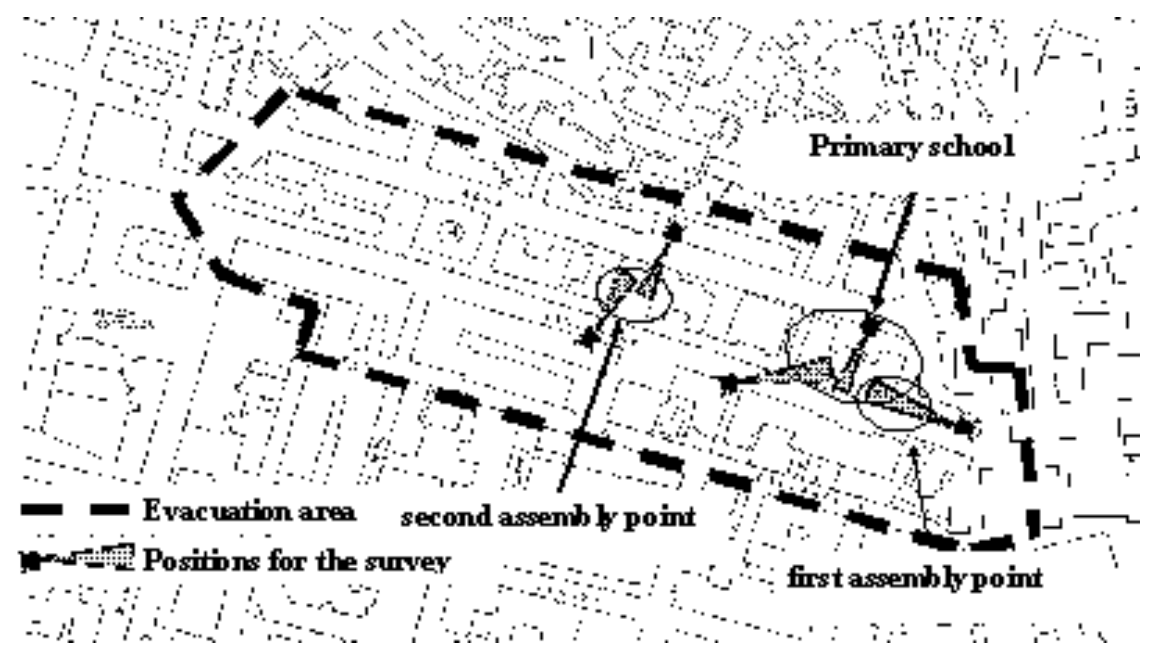

Figure 1: Locations of assembly point and external cameras used for the survey. 


\subsection{Data acquisition}

Prior to experimentation, data was gathered concerning supply (the building plan, evacuation paths) and demand (number of classrooms, school population); during the drill a monitoring system was arranged, with manual/automatic tools and 12 video cameras (external and internal), in order to acquire data concerning pedestrian outflow (times, densities) both inside and outside the building until the gathering places were reached.

The videos obtained (see Fig. 2 for an example of images taken from evacuation videos) were analyzed in the laboratory to extrapolate data needed for model calibration. The time required per flight of steps, from the reference section upstream to the downstream one, was measured for each user and, to identify density, the average number of users on the stairs during this time was counted.

From observations, as also shown in Fig. 2, stairs were used differently. Hence, for the inside staircase, a perceived width equal to half the real one was assumed. In all, 73 users were counted users for the inside stairs and 52 for the external fire escape.

The same data acquisition method was used for boarding time, where from videos both the number of boarding users and the time needed to board were obtained.

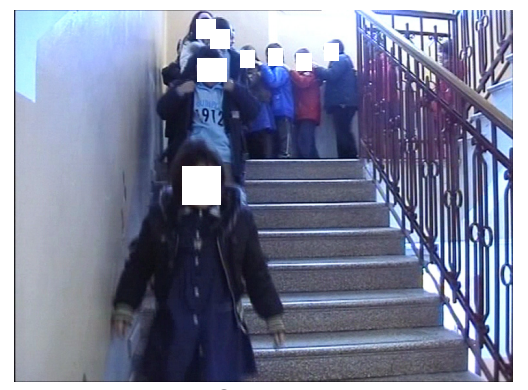

Stairs

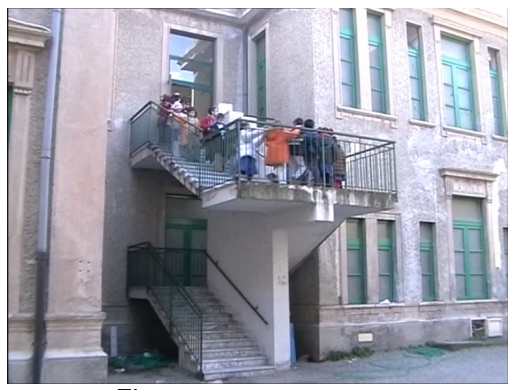

Fire escape

Figure 2: $\quad$ Example of images obtained from the survey.

\subsection{Model calibration and validation}

Starting from eqns. (1) and (2), parameters were calibrated using the Least Squares Method, that is minimizing the sum of square differences between observed values and values provided by the model considered whose parameters have to be known.

Referring to the model expressed by (1), the expression is:

$$
(\mathrm{a}, \mathrm{b}, \mathrm{c}, \mathrm{d}, \mathrm{e}, \mathrm{f})=\underset{\mathrm{a}, \mathrm{b}, \mathrm{c}, \mathrm{d}, \mathrm{e}, \mathrm{f}}{\operatorname{argmin}} \sum_{\mathrm{i}=1, . ., \mathrm{N}}\left(\mathrm{t}_{\mathrm{i}, \mathrm{oss}}-\mathrm{t}_{\mathrm{i}, \mathrm{mod}}\right)^{2}
$$


where:

$\mathrm{N}$ is the number of observations;

$\mathrm{t}_{\mathrm{i}, \text { oss }} \quad$ is the observed value of the time needed for the $\mathrm{i}$-th user to go down the stairs;

$t_{i, \text { mod }}$ is the value estimated by the model of the time needed for the $\mathrm{i}$-th user to go down the stairs.

Calibrations were conducted on a randomly drawn sample of $50 \%$ of observations. Subsequently the calibrated models were validated through an informal test on the calibrated parameter sign and RMSE\% statistic:

$$
\operatorname{RMSE} \%=100 \cdot\left(\left(\sum_{\mathrm{i}=\mathrm{N}}\left(\mathrm{t}_{\mathrm{i}, \text { oss }}-\mathrm{t}_{\mathrm{i}, \mathrm{mod}}\right)^{2}\right) / \mathrm{N}\right)^{1 / 2} /\left(\sum_{\mathrm{i}=\mathrm{N}} \mathrm{t}_{\mathrm{i}, \mathrm{oss}} / \mathrm{N}\right)
$$

Calibration results are shown in Fig. 3. Parameters for boarding time were similarly calibrated.

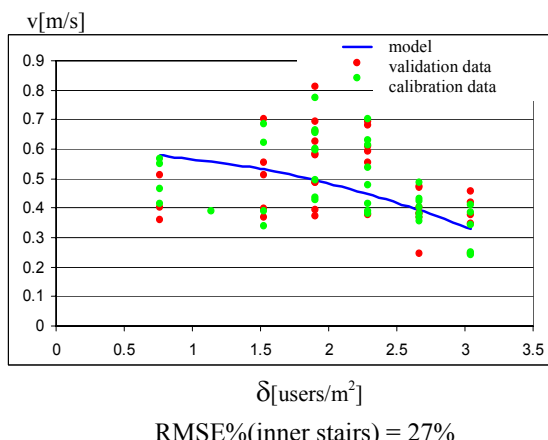

Figure 3: Calibration results.

Table 1: $\quad$ Characteristics of network topology.

\begin{tabular}{lr}
\hline Node classification & nodes \\
\hline origin centroid & 22 \\
destination centroid & 1 \\
real & 99 \\
Total & 122 \\
\hline
\end{tabular}

\begin{tabular}{lr}
\hline Link classification & links \\
\hline fictitious link & 23 \\
corridor & 85 \\
descending flight & 9 \\
Total & 117 \\
\hline
\end{tabular}

\section{Application}

\subsection{Performing simulation}

As an application a computer simulation of the observed evacuation was performed. Referring to the test site, Tab. 1 reports the number of nodes and arcs of the graph to simulate the school evacuation. Paths were obtained from the school evacuation plan. As regards the cost functions adopted, for fictitious links a constant speed function was considered; for corridors we considered a 
relationship between speed and specific flow specified and calibrated in [29]; for descending flights the speed-density functions defined within this paper were considered. Tab. 2 summarizes the cost functions adopted.

Demand values used in the simulation were obtained from school attendance on the experimentation day, and users were located in offices and classrooms following the real distribution. The demand value to be evacuated consists of about 150 users. The first three steps identified in the previous section were simulated with a dynamic approach. The assignment model implemented within the DSS built for this research project allows pedestrian outflow to be simulated with two different hypotheses on distribution of departures:

- departures uniformly distributed in a defined interval;

- departures concentrated at the start of the first simulation interval.

From these considerations, two different kinds of time interval can be defined:

- the time interval within the (distributed) departure, whose length is indicated as LID;

- the time interval between two successive updates of outflow conditions within the simulation model, whose length is given by LIS.

Table 2: $\quad$ Cost functions adopted for the simulation.

\begin{tabular}{cc}
\hline Link & Speed functions \\
\hline $\begin{array}{c}\text { fictitious link } \\
\text { Corridor }\end{array}$ & $\mathrm{v}=$ const. \\
descending flight & $\mathrm{v}=\alpha_{4} \cdot \mathrm{q}_{\mathrm{s}}{ }^{4}+\alpha_{3} \cdot \mathrm{q}_{\mathrm{s}}{ }^{3}+\alpha_{2} \cdot \mathrm{q}_{\mathrm{s}}{ }^{2}+\alpha_{1} \cdot \mathrm{q}_{\mathrm{s}}+\alpha_{0}+\mathrm{P} \cdot(\delta)^{\mathrm{c}}+\mathrm{d} \cdot \mathrm{L}+\mathrm{e} \cdot \mathrm{I} / \mathrm{E}+\mathrm{f} \cdot \mathrm{NG}+\mathrm{g}$ \\
\hline
\end{tabular}

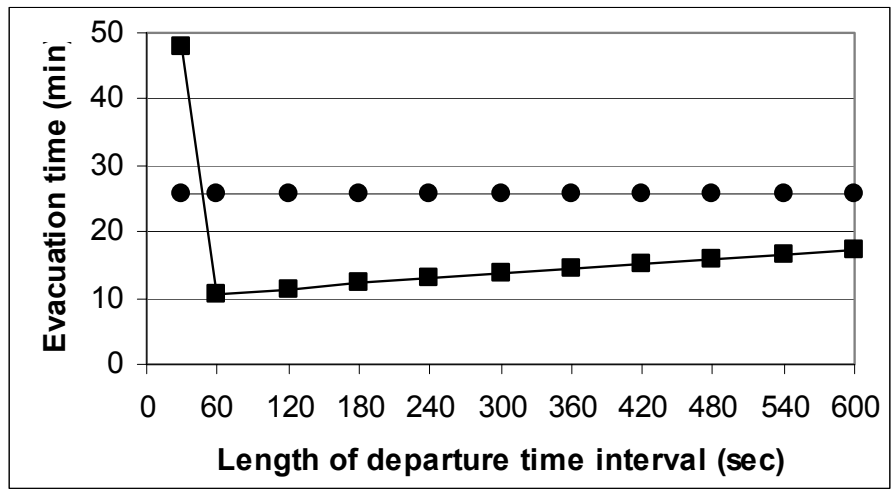

Figure 4: $\quad$ Evacuation time (end of phase 3).

\subsection{Simulation results}

Figure 4 describes the evacuation time up to the second assembly point (the time needed for the last user to reach the second assembly point) depending on LID, considering a fixed value of LIS equal to 30 seconds. It is worth noting that 
evacuation time in the case of concentrated departures remains constant and higher than that obtained with distributed departures.

This happens since users are all introduced into the network at the same time, slowing down at the nodes with lower capacity. In the case of distributed departures, evacuation times increase since users are constrained to put off departures and times are lower since generated outflow conditions avoid queue scattering.

In Tab 3 we report variations obtained by comparing simulated vs. measured components of evacuation time. It can be seen that the computed time can be considered an upper bound of the evacuation time in designing evacuation plans.

Table 3: Analysis of results.

\begin{tabular}{clc}
\hline Phase & \multicolumn{1}{c}{ Description } & Simulated vs. Measured time \\
\hline 1 & Reaching the first assembly point & $36.6 \%$ \\
2 & Waiting at first assembly point & $-3.8 \%$ \\
3 & Reaching the second assembly point & $-14.2 \%$ \\
4 & Boarding on bus & $-0.7 \%$ \\
& Total time & $7.8 \%$ \\
\hline
\end{tabular}

\section{References}

[1] Predetechenskii, V. M. \& Milinskii, A. I., Planning for foot traffic flow in building. Translated from Russian, Amering Publishing co. pvt. Ltd., New Delhi, India, 1978.

[2] Fruin, J.J., Pedestrian planning and design. Metropolitan Association of Urban Designers and Environmental, New York, 1971.

[3] Kendik, E., Assessment of escape routes in building and design method for calculating pedestrian movement. SFPE Technology report 85/4, Society of Fire Protection Engineers, Boston, USA, 1985.

[4] Puschkrev, B. \& Zupan, J., Urban Space for pedestrians. Cambridge, Massachusetts, the MIT press, 1975.

[5] HCM, Highway Capacity Manual. TRB publications, 2000.

[6] Pedestrian and Evacuation Dynamics 2003. In Proceedings of the 2nd International Conference, E.R. Galea (ed).

[7] Russo, F. \& Vitetta, A., Urban road transportation analysis in emergency conditions: models and algorithms. In Proceedings of Urban Transport 2000, Wessex Institute of Technology. Sucharov and Brebbia (eds.), WIT Press Boston, pp. 533-542, 2000.

[8] Russo, F. \& Vitetta, A., Urban transportation system analysis in emergency conditions. In ENVIRONMENTAL HEALTH RISK II, C. A. Brebbia and D. Fayziera (eds), Wit Press, Boston. ISBN 1-85312-983-6, 2003.

[9] Velonà, P. \& Vitetta, A., Evolution of an urban transportation system in emergency conditions: analysis through a pseudo-dynamic assignment 
model. In ENVIRONMENTAL HEALTH RISK II, C. A. Brebbia and D. Fayziera (eds), Wit Press, Boston. ISBN 1-85312-983-6, 2003.

[10] Merchant, D.K. \& Nemhauser, G.L., A model and an algorithm for the dynamic traffic assignment problems. Trans. Sci., 12(3), 183-207, 1978/a.

[11] Merchant, D.K. \& Nemhauser, G.L., Optimal conditions for a dynamic traffic assignment model. In Trans. Sci., 1978/b.

[12] Ben Akiva, M., Cyna, M. \& De Palma, A., Dynamic Model of Peak Period Congestion. In Transportation Research, 1984.

[13] Ben Akiva, M., De Palma, A. \& Kanaraglou P., Dynamic Model of Peak Period Traffic Congestion with Elastic Arrival Rates. In Trans. Sci. 20 (2), 164-181, 1986.

[14] Janson, B. N., Dynamic traffic assignment for urban road networks. In Transportation Research, 1991.

[15] Carey, M., Nonconvexity of the dynamic assignment problem. In Operations Research, 35 No. 5, 58-69, 1992.

[16] Papageorgiou, M. \& Korsialos, A., Short term traffic forecasting with metanet. Daccord Short Term Forecasting Workshop TU Delft, 1998.

[17] Smith, M. J. \& Van Vuren, T., Traffic equilibrium with responsive traffic control. In Trans. Sci., 1993.

[18] Ben Akiva, M., Cascetta, E., Gunn, H., Smulders, S. \& Whittaker, J., DYNA: a real-time monitoring and prediction system for inter-urban motorways. First World Congress on Applications of Transport Telematica and Intelligent Vehicle-Highway Systems. Paris, 1994.

[19] Ben Akiva, M., Bierlaire, M., Bottom, J., Koutsopoulos, H. N. \& Mishalani, R., Development of a route guidance generation system for a real-time application. 8th IFAC/IFIP/IFORS symposium on transportation systems. Chania, Greece, 1997.

[20] Ben Akiva, M., Bierlaire, M., Koutsopoulos, H. N. \& Mishalani, R., DynaMIT: a simulation-based system for traffic prediction and guidance generation. Daccord Short Term Forecasting Workshop TU Delft, 1998.

[21] Cascetta, E. \& Cantarella, G.E., A Day-to-day and Within-day Dynamic Stochastic Assignment Model. Transpn. Res. 25a (5), 277-291, 1991.

[22] Di Gangi, M., Modelling Dynamic Network Loading on Transportation Networks through a Continuous Packet Approach. In Mathematical Modelling of Systems, vol. 2, issue 3, pp.175-196, 1996.

[23] Di Gangi, M., An algorithm for continuous flow Dynamic network loading. VI World Conference on Transportation Research. Lyon, 1992.

[24] Di Gangi M., Modelli a pacchetti per l'assegnazione dinamica alle reti di trasporto. In Metodi e Tecnologie dell'Ingegneria dei Trasporti, Seminario 2002. G.E. Cantarella and F. Russo (eds.) pp. 428-444. Franco Angeli. ISBN 88-464-6181-9, 2005.

[25] Di Gangi, M., Luongo, A. \& Polidoro, R., Una procedura di carico dinamico per la valutazione dei piani di evacuazione. In Metodi $e$ Tecnologie dell'Ingegneria dei Trasporti, Seminario 1999. G.E. Cantarella and F. Russo (eds.) pp. 137-150. Franco Angeli. ISBN 88-4642643-6, 2001. 
[26] Di Gangi, M., Russo, F. \& Vitetta, A., A mesoscopic method for evacuation simulation on passenger ships: models and algorithms. In Pedestrian and Evacuation Dynamics 2003, pp. 197-208 CMS Press, London ISBN 1-904521-08-8, 2003.

[27] Di Gangi, M., Approaching the analysis of transport networks in emergency conditions for the design of evacuation plans. In Risk Analysis $I V$, pp. 485-494, WIT Press. ISBN 1-85312-736-1, 2004.

[28] Vitetta, A., Musolino, G., \& Marcianò, A. F., Safety of users in road evacuation: supply and supply-demand interaction models for users. Third International Conference on Sustainable Development and Planning 2007, 25 -27 April 2007 - Algarve, Portugal, 2007.

[29] Luongo A. S., Le funzioni di deflusso pedonale: aspetti teorici ed applicativi. Università degli Studi della Basilicata, Facoltà di Ingegneria Review of the Dipartimento di Architettura, Pianificazione ed Infrastruttura di Trasporto, special issue devoted to transportation. 